\title{
Nonlinear interactions between upper-hybrid and Alfvén modes in a magnetized plasma containing charged dust impurities
}

\author{
P. K. SHUKLA ${ }^{1,2,3,4,5}$ and L. STEN F L O ${ }^{2}$ \\ ${ }^{1}$ Institut für Theoretische Physik IV and Centre for Plasma Science and Astrophysics, \\ Fakultät für Physik und Astronomie, Ruhr-Universität Bochum, \\ D-44780 Bochum, Germany \\ ${ }^{2}$ Centre for Nonlinear Physics, Department of Physics, Umeå University, \\ SE-90187 Umeå, Sweden \\ ${ }^{3}$ Department of Physics, University of Strathclyde, Glasgow, G4 ONG, UK \\ ${ }^{4}$ Centro de Física dos Plasmas, Departamento de Fisica, Instituto Superior Técnico, \\ Universidade Técnica de Lisboa, 1049-001 Lisbon, Portugal \\ ${ }^{5}$ Centre for Fundamental Physics, Rutherford Appleton Laboratory, \\ Chilton, Didcot, Oxfordshire 0X11 OQX, UK \\ (ps@tp4.rub.de)
}

(Received 23 March 2006 and in revised form 9 May 2006)

\begin{abstract}
We consider the nonlinear interactions between upper-hybrid (UH) and Alfvén modes in a magnetized electron-ion plasma containing a fraction of stationary charged dust grains. The interaction is governed by a pair of equations for the UH wave envelope including the relativistic electron mass increase and the density and compressional magnetic field fluctuations associated with the Alfvén modes that are, in turn, driven by the ponderomotive force of the UH waves. The coupled mode equations are then Fourier analyzed to obtain a new dispersion relation, which admits new classes of modulational instabilities. The existence of a cuspshaped UH envelope soliton is also predicted. The result can have relevance to the electron acceleration by sharply localized UH waves in the dusty magnetosphere of Saturn.
\end{abstract}

\section{Introduction}

Large-amplitude electrostatic upper-hybrid (ES-UH) waves in magnetized plasmas can be excited by electron and photon beams on account of two-stream instabilities and mode conversions. Finite-amplitude ES-UH waves are subjected to a great variety of nonlinear effects [1-10]. About three decades ago, Kaufman and Stenflo [1] considered the nonlinear interactions between ES-UH waves and fast magnetosonic waves propagating across the external magnetic field direction in an electron-ion plasma. They reported the formation of negative group dispersive ES-UH envelope solitons composed of bell-shaped ES-UH wave envelopes and super-magnetosonic density humps in magnetoplasmas with $\omega_{\mathrm{c}}>\omega_{\mathrm{p}} / \sqrt{3}$, where $\omega_{\mathrm{c}}$ and $\omega_{\mathrm{p}}$ are the 
electron gyrofrequency and electron plasma frequency, respectively. Porkolab and Goldman [2] re-examined the ES-UH envelope soliton theory for both negative and positive group dispersions of the UH waves, and presented a modulational instability analysis which revealed that the quasi-stationary modulations of the ESUH waves would lead to the highest growth rates. The coupling between the ES-UH and electrostatic ion-cyclotron perturbations was considered by Yu and Shukla [3] who showed the possibility of a near-sonic cusp-shaped ES-UH envelope soliton. The one-dimensional collapse of ES-UH waves due to a non-local nonlinearity was investigated by Litvak and Sergeev [4]. Berezhiani [5] studied the effect of the relativistic electron mass increase on the ES-UH wave modulation by magnetosonic perturbations. Sharma and Shukla [7], Pécseli [8] and Shukla et al. [9] have also presented comprehensive reviews of the nonlinear effects associated with ES-UH waves involving different types of low-frequency perturbations [10], observed in laboratory experiments $[11,12]$.

Since large-amplitude ES-UH waves are useful for electron acceleration due to the surfatron mechanism [13] in laboratory and astrophysical environments, which may contain a fraction of charged dust impurities, it is of interest to investigate the nonlinear effects associated with ES-UH waves in dusty plasmas [14]. In the latter, we have the possibility of a modified shielding distance [15], new linear and nonlinear wave modes [16-22] and coherent structures $[23,24]$. In the present paper, we revisit the nonlinear interactions between ES-UH waves and modified Alfvén waves (MAWs) [18], accounting for the combined effects of the relativistic electron mass increase in the ES-UH fields and the enhanced density and compressional magnetic field perturbations that are driven by the ES-UH ponderomotive force. We thus extend the work of Shukla and Stenflo [22] by including relativistic effects. When the relativistic nonlinearity cancels a part of the ES-UH group dispersion, new classes of oscillatory modulational instabilities [25] appear. The governing nonlinear equations in a stationary frame admit a cusp-shaped ES-UH envelope soliton when the speed of the latter is close to the modified (by the presence of charged dust grains) Alfvén speed. It is suggested that the localized ES-UH envelope soliton can produce cross-field acceleration of electrons via a surfatron mechanism.

\section{Governing equations}

Let us consider the presence of large-amplitude ES-UH waves in a uniform magnetoplasma composed of electrons, ions and negatively charged dust grains in an external magnetic field $\hat{\mathbf{z}} B_{0}$, where $\hat{\mathbf{z}}$ is a unit vector along the $z$-axis and $B_{0}$ is the strength of the magnetic field. At equilibrium, we have

$$
n_{\mathrm{e} 0}=n_{\mathrm{i} 0}-Z_{\mathrm{d}} n_{\mathrm{d} 0},
$$

where $n_{\mathrm{e} 0}, n_{\mathrm{i} 0}$ and $n_{\mathrm{d} 0}$ are the unperturbed electron, ion and dust number densities, respectively, and $Z_{\mathrm{d}}$ is the number of electrons residing on a stationary dust particle. The nonlinear coupling between perpendicularly (to $\hat{\mathbf{z}}$ ) propagating ES-UH waves with the compressional MAW perturbations in a magnetized dusty plasma is governed by the ES-UH envelope equation [9]

$$
2 i \omega_{\mathrm{H}}\left(\frac{\partial}{\partial t}+V_{\mathrm{g}} \frac{\partial}{\partial x}\right) E_{x}+3 \alpha V_{\mathrm{Te}}^{2} \frac{\partial^{2} E_{x}}{\partial x^{2}}+\Omega_{0}^{2} \frac{\left|E_{x}\right|^{2}}{E_{c}^{2}} E_{x}-\Omega_{0}^{2} N E_{x}=0
$$


and the driven MAW equation [22]

$$
\left(\frac{\partial^{2}}{\partial t^{2}}+\Omega_{\mathrm{R}}^{2}-V_{\mathrm{AR}}^{2} \frac{\partial^{2}}{\partial x^{2}}\right) N=\frac{m_{\mathrm{e}} n_{\mathrm{i} 0}}{3 m_{\mathrm{i}} n_{\mathrm{e} 0}} \frac{c^{2}}{E_{c}^{2}} \frac{\partial^{2}\left|E_{x}\right|^{2}}{\partial x^{2}},
$$

where $\omega_{\mathrm{H}}=\left(\omega_{\mathrm{p}}^{2}+\omega_{\mathrm{c}}^{2}\right)^{1 / 2}$ is the upper-hybrid resonance frequency, $V_{\mathrm{g}}=3 k_{0} \alpha V_{\mathrm{Te}}^{2} / \omega_{\mathrm{H}}$ is the ES-UH wave group velocity, $k_{0}$ is the wavenumber, $\alpha=\left(1-3 \omega_{\mathrm{c}}^{2} / \omega_{\mathrm{p}}^{2}\right)^{-1}$, $V_{\mathrm{Te}}$ is the electron thermal speed, $\Omega_{0}=\left(\omega_{\mathrm{p}}^{2}+2 \omega_{\mathrm{c}}^{2}\right)^{1 / 2}, E_{c}=2 m_{\mathrm{e}} c \omega_{\mathrm{p}}^{2} / \sqrt{3} e \omega_{\mathrm{H}}, m_{\mathrm{e}}$ is the electron rest mass, $c$ is the speed of light in vacuum, $e$ is the magnitude of the electron charge and $N=n_{\mathrm{e} 1} / n_{\mathrm{e} 0}\left(=B_{1 z} / B_{0}\right) \ll 1$ is the normalized electron number density perturbation (compressional magnetic field) associated with the MAWs in dusty plasmas. The $x$-component (transverse to $\hat{\mathbf{z}}$ ) of the ES-UH electric field is denoted by $E_{x}$. We see that the ES-UH waves have negative group dispersion for $\omega_{\mathrm{p}}<\sqrt{3} \omega_{\mathrm{c}}$. The fourth term on the left-hand side of (1) comes from the relativistic electron mass increase, while the fifth term is associated with the density and compressional magnetic field perturbations that are reinforced by the ES-UH wave ponderomotive force [2]. Furthermore, $\Omega_{\mathrm{R}}=Z_{\mathrm{d}} n_{\mathrm{d} 0} \omega_{\mathrm{ei}} / n_{\mathrm{e} 0}$ is the Rao cut-off frequency [18], $V_{\mathrm{AR}}=\left(n_{\mathrm{i} 0} / n_{\mathrm{e} 0}\right) B_{0} / \sqrt{4 \pi n_{\mathrm{i} 0} m_{\mathrm{i}}}$ is the modified Alfvén speed [18] in a magnetized electron-ion-dust plasma, where $\omega_{\mathrm{ci}}=e B_{0} / m_{\mathrm{i}} c$ is the ion gyrofrequency and $m_{\mathrm{i}}$ is the ion mass.

It should be mentioned that (2) has been derived by invoking the slowly varying envelope approximation (viz. $\partial E_{x} / \partial t \ll \omega_{\mathrm{H}} E_{x}$ ) on the ES-UH wave equation that is obtained by combining the linearized electron continuity and momentum (with an appropriate pressure term to accommodate the negative group dispersion of the ES-UH wave) equations together with the Poisson equation. In the ES-UH wave equation, we have also included the relativistic electron mass increase nonlinearity, as well as the electron density and compressional magnetic field perturbations (viz. $n_{\mathrm{e} 1}$ and $B_{1 z}$ ) associated with the MAWs [22]. The latter obey (2), which is obtained from a combination of the Faraday and Ampére laws together with the ion continuity equation and the ion momentum equation in which the electric field was eliminated by the inertialess electron equation of motion that contained the $x$-component of the ES-UH wave ponderomotive force [2]. The latter arises owing to the averaging of the $x$-component of the electron advection nonlinearity over one period of the ES-UH waves.

It is convenient to denote $E=E_{x} / E_{c}$ and rewrite the pair of equations (2) and (3) as

$$
2 i \omega_{\mathrm{H}}\left(\frac{\partial}{\partial t}+V_{\mathrm{g}} \frac{\partial}{\partial x}\right) E+3 \alpha V_{\mathrm{Te}}^{2} \frac{\partial^{2} E}{\partial x^{2}}+\Omega_{0}^{2}\left(|E|^{2}-N\right) E=0
$$

and

$$
\left(\frac{\partial^{2}}{\partial t^{2}}+\Omega_{\mathrm{R}}^{2}-V_{\mathrm{AR}}^{2} \frac{\partial^{2}}{\partial x^{2}}\right) N=\frac{m_{\mathrm{e}} n_{\mathrm{i} 0}}{3 m_{\mathrm{i}} n_{\mathrm{e} 0}} c^{2} \frac{\partial^{2}|E|^{2}}{\partial x^{2}} .
$$

Equations (4) and (5) are the desired set for studying the modulational instability and collapse of the ES-UH wave packets in a magnetized electron-ion-dust plasma.

\section{Nonlinear dispersion relation and localized ES-UH envelope solution}

In order to investigate the modulational instability of a constant-amplitude ES-UH pump $E_{x 0} \exp \left(-i \omega_{\mathrm{H}} t+i k_{0} x\right)+$ complex conjugate, we decompose $E_{x}$ as the sum 
of the pump and its two sidebands. Hence, following the standard procedure [9] for deriving the nonlinear dispersion relation, we have, from (4) and (5),

$$
\begin{gathered}
\left(\Omega^{2}-\Omega_{\mathrm{AR}}^{2}\right)\left[\left(\Omega-K V_{\mathrm{g}}\right)^{2}-\frac{3 \alpha K^{2} V_{\mathrm{Te}}^{2}}{4 \omega_{\mathrm{H}}^{2}}\left(3 \alpha K^{2} V_{\mathrm{Te}}^{2}-\Omega_{0}^{2} W_{0}^{2}\right)\right] \\
=\frac{\alpha K^{2} V_{\mathrm{Te}}^{2} m_{\mathrm{e}} n_{\mathrm{i} 0}}{4 m_{\mathrm{i}} n_{\mathrm{e} 0} \omega_{\mathrm{H}}^{2}} \Omega_{0}^{2} K^{2} c^{2} W_{0}^{2}
\end{gathered}
$$

where $\Omega$ and $K$ are the frequency and wavenumber of the MAWs, $\Omega_{\mathrm{AR}}=\left(\Omega_{\mathrm{R}}^{2}+\right.$ $\left.K^{2} V_{\mathrm{AR}}^{2}\right)^{1 / 2}$ and $W_{0}=\left|E_{x 0}\right| / E_{c}$. The nonlinear dispersion relation (6) admits interesting solutions when $\alpha>0, \sqrt{3 \alpha} K V_{\mathrm{Te}} \sim \Omega_{0} W_{0}$ and $\Omega \ll \Omega_{\mathrm{AR}}$. Then we have

$$
\Omega=K V_{\mathrm{g}} \pm i \sqrt{\alpha \delta} \frac{\Omega_{0} K V_{\mathrm{Te}}}{2 \Omega_{\mathrm{AR}} \omega_{\mathrm{H}}} K c W_{0},
$$

which depicts an oscillatory modulational instability. Here we have introduced $\delta=$ $m_{\mathrm{e}} n_{\mathrm{i} 0} / m_{\mathrm{i}} n_{\mathrm{e} 0}$. Furthermore, for $\alpha>0, \sqrt{3 \alpha} K V_{\mathrm{Te}} \sim \Omega_{0} W_{0}$ and $\Omega \gg \Omega_{\mathrm{AR}}$ we have, from (6),

$$
\Omega=\frac{1}{2} K V_{\mathrm{g}} \pm \frac{1}{2} \sqrt{K^{2} V_{\mathrm{g}}^{2} \pm 2 \Omega_{\mathrm{D}}^{2}}
$$

where $\Omega_{\mathrm{D}}^{2}=\sqrt{\alpha \delta} K V_{\mathrm{Te}} \Omega_{0} K c W_{0} / \omega_{\mathrm{H}}$, which also predicts an oscillatory modulational instability.

Let us now present possible stationary solutions of (4) and (5). We then seek solutions of the form $E=\mathscr{E}\left(\xi=x-V_{\mathrm{g}} t\right) \exp (i \Theta(t))$ and $N=N(\xi)$, with $V_{\mathrm{g}} \sim V_{\mathrm{AR}}$. Hence, we have from (5) that

$$
N=\frac{\delta c^{2}}{3 \Omega_{\mathrm{R}}^{2}} \frac{\partial^{2} \mathscr{E}^{2}}{\partial \xi^{2}} .
$$

Inserting the non-local density response (9) into (4) and invoking stationarity in the moving frame $x-V_{\mathrm{g}} t$, we obtain

$$
3 \alpha V_{\mathrm{Te}}^{2} \frac{\partial^{2} \mathscr{E}}{\partial \xi^{2}}-\Omega^{2} \mathscr{E}+\Omega_{0}^{2} \mathscr{E}\left(\mathscr{E}^{2}-\lambda^{2} \frac{\partial^{2} \mathscr{E}^{2}}{\partial \xi^{2}}\right)=0
$$

where $\Omega=\left(2 \omega_{\mathrm{H}} \partial \Theta / \partial t\right)^{1 / 2}$ and $\lambda=\sqrt{\delta} c / \sqrt{3} \Omega_{\mathrm{R}}$. Equation (10) is a nonlinear Schrödinger equation containing both cubic and non-local nonlinearities. For $\alpha>0$ it admits a cusp-shaped electric field profile [4] of the ES-UH waves trapped in an electron density cavity, which has shoulders on its sides.

\section{Summary}

In this paper, we have re-examined the nonlinear coupling between the ES-UH waves and MAWs in an electron-ion-dust magnetoplasma, taking into account the combined effects of the relativistic electron mass increase and the influence of the ES-UH wave ponderomotive force on the MAWs. Specifically, the model of Shukla and Stenflo [22] has been extended to include the relativistic nonlinearity in the ES-UH envelope equation. Our new pair of equations is then Fourier analyzed to obtain a nonlinear dispersion relation. The latter exhibits a new class of 
oscillatory modulational instabilities for the case in which the relativistic electron mass increase nonlinearity cancels a part of the UH wave group dispersion. Possible stationary solutions of our present nonlinear equations, comprising cubic and nonlocal nonlinearities in the Schrödinger equation, can be represented in the form of spiky ES-UH electric fields trapped locally in an electron density cavity having shoulders on its sides. The results presented here are essential for understanding the nonlinear instability of the ES-UH waves and the associated localized UH wave packets that can accelerate electrons across the magnetic field direction via a surfatron acceleration mechanism. Such a scenario could occur in the dusty magnetosphere of Saturn, where ES-UH waves have been observed by the Cassini spacecraft [26].

\section{Acknowledgements}

This work was partially supported by the Deutsche Forschungsgemeinschaft through the Sonderforschungsbereich 591 entitled 'Universelles Verhalten gleichgewichtsferner Plasmen: Heizung Transport und Strukturbildung', and by the Swedish Research Council.

\section{References}

[1] Kaufman, A. N. and Stenflo, L. 1975 Phys. Scripta 11, 269.

[2] Porkolab, M. and Goldman, M. V. 1976 Phys. Fluids 19, 872.

[3] Yu, M. Y. and Shukla, P. K. 1977 Phys. Plasma 19, 889.

[4] Litvak, A. G. and Sergeev, A. M. 1978 JETP Lett. 27, 517.

[5] Berezhiani, V. I. 1981 Sov. J. Plasma Phys. 7, 365.

[6] Murtaza, G. and Shukla, P. K. 1984 J. Plasma Phys. 31, 423.

[7] Sharma, R. P. and Shukla, P. K. 1983 Phys. Plasmas 26, 87.

[8] Pécseli, H. 1985 IEEE Trans. Plasma Sci. PS-13, 53.

[9] Shukla, P. K., Rao, N. N., Yu, M. Y. and Tsintsadze, N. L. 1986 Phys. Rep. 138, 1.

[10] Yu, M. Y. and Shukla, P. K. 1978 J. Math. Phys. 19, 2506; 1983 J. Plasma Phys. 29, 409.

[11] Christiansen, P. J., Jain, V. K. and Stenflo, L. 1981 Phys. Rev. Lett 46, 1333.

[12] Cho, T. and Tanaka, S. 2001 Phys. Rev. Lett. 45, 1403.

[13] Katsouleas, T. and Dawson, J. M. 1983 Phys. Rev. Lett. 51, 392. Chernikov, A. A., Schmidt, G. and Neishtadt, A. 1992 Phys. Rev. Lett. 68, 1507.

[14] Shukla, P. K. and Mamun, A. A. 2002 Introduction to Dusty Plasma Physics. Bristol: IoP.

[15] Shukla, P. K. 1994 Phys. Plasmas 1, 1362.

[16] Rao, N. N., Shukla, P. K. and Yu, M. Y. 1990 Planet. Space Sci. 38, 543. Shukla, P. K. 1992 Phys. Scripta 45, 504.

Shukla, P. K. and Silin, V. 1992 Phys. Scripta 45, 508.

Shukla, P. K. 2001 Phys. Plasmas 8, 1791.

[17] Varma, R. K., Shukla, P. K. and Krishan, V. 1993 Phys. Rev. E 47, 3612.

[18] Rao, N. N. 1995 J. Plasma Phys. 53, 317.

[19] Birk, G. T., Kopp, A. and Shukla, P. K. 1996 Phys. Plasmas 3, 3564.

[20] Amin, M. R., Morfill, G. E. and Shukla, P. K. 1998 Phys. Rev. E 59, 6517. Shukla, P. K. 2000 Phys. Plasmas 7, 1044.

[21] Yu, M. Y. and Shukla, P. K. 1983 J. Plasma Phys. 29, 409. Shukla, P. K., Birk, G. T. and Bingham, R. 1995 Geophys. Res. Lett. 22, 671. Popel, S. I., Vladimirov, S. V. and Shukla, P. K. 1995 Phys. Plasmas 2, 716. 
[22] Shukla, P. K. and Stenflo, L. 2003 Phys. Plasmas 10, 4572.

[23] Pokhotelov, O. A., Onishchenko, O. G., Shukla, P. K. and Stenflo, L. 1999 J. Geophys. Res. 104, 19797.

[24] Mamun, A. A. and Shukla, P. K. 2002 Phys. Plasmas 9, 1468; 2001 Phys. Lett. A 290, 173.

[25] Tsintsadze, N. L., Tskhakaya, D. D. and Stenflo, L. 1979 Phys. Lett. A 72, 115.

[26] Williams, J. D., Chen, L. J., Kurth, W. S., Gurnett, D. A. and Dougherty, M. K. 2006 Geophys. Res. Lett. 33, L06103/1-4; doi: 10.1029/2005GL024532. 